\title{
Urgent-Start Peritoneal Dialysis - a viable option? A case report and literature review
}

\author{
Joana Tavares ${ }^{1}$, Filipa Silva ${ }^{1}$, Anna Lima ${ }^{2}$, Maria João Carvalho ${ }^{1}$, António Cabrita ${ }^{1}$, Anabela Rodrigues ${ }^{1}$ \\ ${ }^{1}$ Serviço de Nefrologia do Centro Hospitalar Universitário do Porto. Largo do Prof. Abel Salazar 4099-001 Porto, Portugal \\ ${ }^{2}$ Serviço de Nefrologia do Hospital Prof. Doutor Fernando Fonseca - EPE. IC 19, 2720-276 Amadora, Portugal
}

\section{ABSTRACT}

Background: Many patients with end-stage renal disease start renal replacement therapy in an unplanned manner. The vast majority initiate hemodialysis by a central venous catheter, since its use is more widespread and available. This technique is associated with a high risk of infection and damage of the vascular patrimony associated with the use of central veins. Urgent-start peritoneal dialysis comes as an alternative treatment for selected patients. Case report: A 55-year-old woman with focal segmental glomerulosclerosis presented with a rapid decline of renal function and was given renal replacement therapy counselling and opted for peritoneal dialysis. Her chosen modality was postponed for one month due to early uremic symptoms, followed by hemodialysis start through a central venous catheter. During this period a sepsis due to central venous catheter infection occurred, implying four weeks of intravenous antibiotics. Discussion and Conclusion: Although there has been an increase in the number of publications on urgent-start peritoneal dialysis, showing that this technique has comparable results either to urgent-start hemodialysis and planned-start peritoneal dialysis, there still is some resistance to the use of this modality. Given the importance of this subject, this review aims to describe and summarize the available evidence on urgent-start peritoneal dialysis outcomes. Moreover, specific barriers are addressed. Its use is encouraged in hospitals where peritoneal dialysis is available, as an opportunity to improve chronic kidney disease patient management and transition to dialysis.

Keywords: End-stage renal disease; Peritoneal Dialysis; Renal replacement therapy; Urgent-start dialysis.

\section{BACKGROUND}

Portugal has one of the highest incidence and prevalence of renal replacement therapy in the world. Worldwide, the incidence and prevalence of the Portuguese dialysis population ranks $5^{\text {th }}$ and $9^{\text {th }}$, respectively ${ }^{1}$.

According to the Portuguese Registry of Dialysis and Transplantation in 2018, not only has the prevalence increased proportionally throughout time, but the last twenty years have seen the highest-ever numbers of patients admitted to dialysis ${ }^{2}$.

The majority (>90\%) of Portuguese patients suffering from ESRD undergo hemodialysis (HD) as their first renal replacement therapy (RRT). In contrast, less than $10 \%$ begin peritoneal dialysis $(P D)^{3,4}$.

Moreover, around half of the patients initiate dialysis with no planned dialysis access, due to late referral or unexpected acute exacerbation of the disease ${ }^{5,6}$.

This situation is illustrated by the Portuguese numbers registered in 2018 , where $58.9 \%$ of the patients started dialysis through a central venous catheter ( $51.6 \%$ via a tunneled catheter and $7.3 \%$ a temporary catheter $)^{2}$.

The downside of this practice is that the use of catheters in unplanned dialysis start has been listed as an independent risk factor of mortality, since it is associated with a higher number of infectious complications ${ }^{7}$. Moreover, after starting HD, most patients remain on this technique, as PD is rarely presented once the treatment is established $^{8}$.

Urgent-start PD, defined as dialysis initiation within two weeks of catheter placement, has been accepted as a viable and safe practice for unplanned dialysis start ${ }^{9-11}$.

\section{CASE REPORT}

A 55-year-old female, with a biopsy proven focal segmental glomerulosclerosis (FSGS), presented with a nephrotic syndrome refractory to multiple therapies. She rapidly evolved to end-stage renal disease within one year and PD was her choice for RRT. Unfortunately, before it was possible to plan PD start, the patient developed the 
uremic symptoms of anorexia, nausea, pruritus and had anemia and hyperphosphatemia.

\section{What to do next?}

Figure 1 describes the advantages of each technique initiation.

\section{Figure 1}

Advantages of $\mathrm{HD}$ and $\mathrm{PD}$ in an unplanned setting

\section{HD}

\section{PD}

- CVC widely available in $24 \mathrm{~h}$

- Faster volume control as well as faster correction of hydro electrolyte and acid-base disturbances.

- Prompt discharge to outpatient clinic

- Shared use with other techniques if needed (e.g.: plasmapheresis)

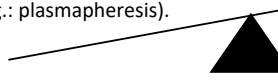

A tunneled central venous catheter (CVC) was placed and hemodialysis was started.

Two weeks later, she presented fever and hypotension during dialysis sessions, and the diagnosis of sepsis due to CVC infection was presumed. Blood cultures were collected and empiric therapy with vancomycin and ceftazidime was started. She rapidly showed signs of clinical and analytical improvement. However, no microorganism was identified, and both antibiotics were maintained for four weeks. Once the infection was resolved, a peritoneal catheter was placed and one month later, she started continuous ambulatory peritoneal dialysis (CAPD).

\section{DISCUSSION}

In this present case, it is inevitable to wonder that had the patient initially started PD, as she opted to do, one severe infection and the use of a central venous access could have been avoided.

With the improvement of the healthcare provided to end-stage renal patients comes increased life expectancy, which means that patients live longer and switch from one technique to the other. Accordingly, we should manage a patient considering the entirety of his or her pathway and the possible benefit of the transitions track, rather than focusing only on the actual modality at the time of our observation. With this goal in mind, why not consider, should the candidate be suitable, even in unplanned situations, a technique that offers protection of the vascular patrimony, protection of the residual renal function and, more importantly, protection of the patient throughout the disease course, as PD does? In fact, short-term survival on PD is benefited by the avoidance of central catheters and long-term survival is similar to HD, but more than survival, relevant outcomes such as sparing vessels and protecting residual renal function should also be taken as clinical priorities in CKD management.

\section{Literature review}

Since the beginning of the millennium, there has been mounting evidence of the feasibility of an urgent-start PD program. Tables I to III summarize the main characteristics of these studies. Most of these studies vary in terms of methods and population but the endpoints are quite similar, evaluating mechanical and infectious complications, as well as patient and technique survival. Urgent-start intervention varies from immediate start until 2 weeks after implantation. Incidence of leakage occurs in 1-10\% of the patients and catheter dysfunction in $2.4-12 \%$ of the patients ${ }^{12-19}$. The patient and technique survival rates are high, $80-100 \%$ and $70-90 \%$ respectively $12,14,16,18,19$. Wong et al., compared the results of different techniques of catheter placement in urgent-start PD programs: percutaneous, laparoscopic or surgical procedure are viable methods ${ }^{18}$. Recently Wang et al., showed that both intermittent and automatic peritoneal dialysis are suitable in an urgent setting ${ }^{17}$.

\section{Urgent-start PD vs. Planned-start PD}

In comparing unplanned to planned start of $P D$, the number of infectious complications does not differ significantly ${ }^{8,20-24}$. Concerning mechanical complications, Povlsen and Ivarsen reported an overall increase of mechanical complications, in a study including 140 patients. However, when analyzing each complication, leakage and catheter dysfunction, separately, there were no statistically significant differences ${ }^{22}$. Later, in 2012 Ghafari et al. reported in a 27 patient-study an increase of the percentage of leakage $(p<0.05)$ in the urgent-start group, but most times it could be managed conservatively, and did not affect the survival of patient in the technique ${ }^{8}$. Patient survival rates were also similar between the two groups ${ }^{8,20-23}$.

When it comes to dialysis efficiency, little data is available, but differences in analytical parameters such as $K t / V$, hemoglobin, iron saturation, parathyroid hormone, calcium, phosphorus and albumin values in urgent-start PD and planned-start PD groups, were not reported $^{8}$.

\section{Urgent-start PD vs. Urgent-start HD}

Other studies have shown similar outcomes between urgent-start PD and urgent-start HD in terms of survival, despite significant differences in infection risk. Koch et al. in 2012 showed a higher risk for bacteremia in the HD group, when comparing both techniques in a 123 patient-study. In addition, Jin et al. in 2016 presented that not only there was a higher overall infectious rate in the HD group $(p=0.014)$ but there was also an increase of overall non-infectious complications in this group $(p=0.001)$. Survival rates were similar in both modalities of treatment ${ }^{9-11}$.

However, irrespective of the dialytic treatment option, it seems that unplanned start is associated with a worse outcome, possibly due to uremic syndrome and other comorbidities ${ }^{7}$.

Considering urgent-start PD a feasible technique for unplanned situations, it is vital to widen the use of PD for crashing patients. This is critical in patients who have chosen PD as the modality of chronic RRT and who need urgent-start dialysis. In these patients, efforts 
$\underline{\text { Table I }}$

Observational studies into Urgent-Start PD

\begin{tabular}{|c|c|c|c|c|c|c|c|}
\hline Study & Patients (n) & Catheter placement & $\begin{array}{l}\text { Urgent-start } \\
\text { Intervention }\end{array}$ & Follow-up & Mechanical Complications & Infectious Complications & Patient and technique surviva \\
\hline $\begin{array}{l}\text { Song et al., } \\
2000\end{array}$ & 59 & Percutaneous & $\begin{array}{l}\text { Immediate start } \\
\text { (<24h): (I) Gradual } \\
\text { increase vs. (II) Full } \\
\text { 2L exchange }\end{array}$ & 12 months & $\begin{array}{l}\text { Leakage: } 9.5 \% \text { (I) vs. } 10.5 \% \text { (II) } \\
\text { [NS] } \\
\text { Catheter dysfunction: } 4.8 \% \text { (I) } \\
\text { vs. } 5.3 \% \text { (II) [NS] }\end{array}$ & $\begin{array}{l}\text { Peritonitis: } 24 \% \text { (I) vs. } 16 \% \text { (II) } \\
\text { [NS] } \\
\text { ESI: } 9.5 \% \text { (I) vs. } 5.3 \% \text { (II) [NS] }\end{array}$ & $\begin{array}{l}\text { Catheter survival: } 86 \% \text { (I) vs. } \\
84 \% \text { (II) [NS] }\end{array}$ \\
\hline $\begin{array}{l}\text { Banli et al., } \\
2005\end{array}$ & 41 & Percutaneous & $\begin{array}{l}\text { Gradual start } 6 \text { days } \\
\text { after implantation }\end{array}$ & NA & $\begin{array}{l}\text { Leakage: } 4.8 \% \\
\text { Catheter dysfunction: } 2.4 \%\end{array}$ & $\begin{array}{l}\text { Peritonitis: } 2.4 \% \\
\text { ESI: } 0 \%\end{array}$ & Not evaluated \\
\hline $\begin{array}{l}\text { Jo et al., } \\
2007\end{array}$ & 51 & Percutaneous & $\begin{array}{l}\text { Immediate start } \\
(<24 \mathrm{~h})\end{array}$ & 12 months & $\begin{array}{l}\text { Leakage: } 2 \% \\
\text { Catheter dysfunction: } 12 \%\end{array}$ & $\begin{array}{l}\text { Peritonitis: } 4 \% \\
\text { ESI: } 4 \%\end{array}$ & Not evaluated \\
\hline $\begin{array}{l}\text { Alkatheeri } \\
\text { et al., } 2016\end{array}$ & 30 & $\begin{array}{l}\text { Percutaneous } \\
\text { (67\%) or } \\
\text { laparoscopic (33\%) }\end{array}$ & $\begin{array}{l}\text { Start in a median of } \\
6 \text { days }\end{array}$ & $\begin{array}{l}\text { Median of } \\
201 \text { days }\end{array}$ & $\begin{array}{l}\text { Leakage: } 10 \% \\
\text { Catheter dysfunction: } 20 \%\end{array}$ & $\begin{array}{l}\text { Peritonitis: 1: } 319 \text { patient-year } \\
\text { ESI: 1:159 patient-year }\end{array}$ & $\begin{array}{l}\text { Patient survival: } 100 \% \\
\text { Technique survival: } 93 \%\end{array}$ \\
\hline $\begin{array}{l}\text { Wong et } \\
\text { al., } 2016\end{array}$ & 81 & $\begin{array}{l}\text { Either } \\
\text { percutaneous, } \\
\text { laparoscopic or } \\
\text { surgical }\end{array}$ & $\begin{array}{l}\text { Urgent start (before } \\
2 \text { weeks) }\end{array}$ & 12 months & $\begin{array}{l}\text { Leakage: } 5 \% \\
\text { Catheter dysfunction: } 15 \%\end{array}$ & Peritonitis: $16 \%$ & $\begin{array}{l}\text { Patient survival: } 100 \% \\
\text { Technique survival: } 74 \%\end{array}$ \\
\hline $\begin{array}{l}\text { Bitencourt } \\
\text { Dias et al., } \\
2017\end{array}$ & 51 & Percutaneous & $\begin{array}{l}\text { Start in } 72 \mathrm{~h} \text { with } \\
\text { high-volume PD }\end{array}$ & 6 months & $\begin{array}{l}\text { Leakage: } 9.7 \% \\
\text { Catheter migration: } 16 \%\end{array}$ & $\begin{array}{l}\text { Peritonitis: } 0,5 \text { patient-year } \\
\text { ESI: } 17 \%\end{array}$ & $\begin{array}{l}\text { Patient survival: } 82.4 \% \\
\text { Technique survival: } 86 \%\end{array}$ \\
\hline $\begin{array}{l}\text { Xu et al., } \\
2017\end{array}$ & 922 & Surgical & $\begin{array}{l}\text { Start In the } 1^{\text {st }} \\
\text { week }\end{array}$ & $\begin{array}{l}\text { Median of } \\
31 \text { months }\end{array}$ & $\begin{array}{l}\text { Abdominal wall related: } 4.8 \% \\
\text { (mainly hernia) } \\
\text { Leakage: } 1 \% \\
\text { Catheter related: } 9.5 \%\end{array}$ & Not evaluated & Technique survival: 92\% \\
\hline $\begin{array}{l}\text { Wang et } \\
\text { al., } 2017\end{array}$ & 101 & Surgical & $\begin{array}{l}\text { Start } 2 \text { days after. } \\
\text { Intermittent group } \\
\text { (IPD): } 50 \% \\
\text { Automatic group } \\
\text { (APD): } 50 \%\end{array}$ & 12 months & $\begin{array}{l}\text { Leakage: } 10 \% \text { (IPD) vs. } 3.9 \% \\
\text { (APD) [NS] } \\
\text { Catheter malposition: } 4 \% \\
\text { (IPD) vs. } 3.9 \% \text { (APD) [NS] }\end{array}$ & $\begin{array}{l}\text { Infections: 26\% (IPD) and } \\
13.7 \% \text { (APD) [NS] }\end{array}$ & Not evaluated \\
\hline
\end{tabular}

APD - Automatic peritoneal dialysis; ESI - Exit site infection; HD - Hemodialysis; IPD - Intermittent peritoneal dialysis; NA - Not applied; NS - No significance; PD - Peritoneal Dialysis; PL - planned; US - Urgent start.

$\underline{\text { Table II }}$

Urgent-start PD versus Planned-start PD

\begin{tabular}{|c|c|c|c|c|c|c|c|}
\hline Study & Patients (n) & Catheter placement & $\begin{array}{l}\text { Urgent-Start } \\
\text { Intervention }\end{array}$ & Follow-up & Mechanical Complications & Infectious Complications & Survival \\
\hline $\begin{array}{l}\text { Povlsen } \\
\text { and } \\
\text { Ivarsen, } \\
2006\end{array}$ & $\begin{array}{l}\text { 140: } 52 \\
\text { (US) vs. } 88 \\
\text { (PS) }\end{array}$ & Surgical & $\begin{array}{l}\text { Immediate start } \\
\text { (<24h) }\end{array}$ & 3 months & $\begin{array}{l}\text { Overall: } 28.9 \% \text { (US) vs. } 7.7 \% \\
\text { (PS), p }<0.01 \\
\text { Leakage and } \\
\text { catheter dysfunction: [NS] }\end{array}$ & $\begin{array}{l}\text { Peritonitis: } 15 \% \text { (US) vs. } 15 \% \\
\text { (PS) [NS] } \\
\text { ESI: } 3.9 \% \text { (US) vs. 3,9\% (PS) } \\
\text { [NS] }\end{array}$ & $\begin{array}{l}\text { Technique survival: } 87 \% \text { (US) } \\
\text { vs. 90\% (PS) [NS] }\end{array}$ \\
\hline $\begin{array}{l}\text { Yang et al., } \\
2011\end{array}$ & $\begin{array}{l}\text { 310: } 226 \\
\text { (US) vs. } 84 \\
\text { (PS) }\end{array}$ & Surgical & $\begin{array}{l}\text { Urgent start (before } \\
2 \text { weeks); } \\
\text { Continuous } \\
\text { ambulatory PD. }\end{array}$ & 6 months & $\begin{array}{l}\text { Leakage: } 2.2 \% \text { (US) vs. } 2.4 \% \\
\text { (PS) [NS] } \\
\text { Catheter dysfunction: } 1.3 \% \\
\text { (US) vs. } 0 \% \text { (PS) [NS] }\end{array}$ & $\begin{array}{l}\text { Peritonitis: } 4 \% \text { (US) vs. } 2.3 \% \\
\text { (PS) [NS] } \\
\text { ESI: } 1.3 \% \text { (US) vs. } 0 \% \text { (PS) [NS] }\end{array}$ & $\begin{array}{l}\text { Not possible to be compared } \\
\text { due to significant differences } \\
\text { in the follow-up time between } \\
\text { the groups. }\end{array}$ \\
\hline $\begin{array}{l}\text { Ghafari, } \\
2012\end{array}$ & $\begin{array}{l}\text { 27: } 18 \text { (US) } \\
\text { vs. } 9 \text { (PS) }\end{array}$ & Percutaneous & $\begin{array}{l}\text { Urgent start (before } \\
2 \text { weeks) }\end{array}$ & 3 months & $\begin{array}{l}\text { Leakage: } 33 \% \text { (US) vs. } 11 \% \\
\text { (PS), p< } 0.05 \\
\text { Catheter dysfunction: } 11 \% \\
\text { (US) vs. } 22 \% \text { (PS) [NS] }\end{array}$ & $\begin{array}{l}\text { Peritonitis: } 1 / 110 \text { patient- } \\
\text {-month (US) vs. } 1 / 42 \text { patient- } \\
\text {-month (PS) [NS] } \\
\text { ESI: } 1 / 55 \text { patient-month (US) } \\
\text { vs. } 1 / 42 \text { patient-month (PS) } \\
\text { [NS] }\end{array}$ & $\begin{array}{l}\text { Loss of follow-up. From the } \\
\text { data available: } \\
\text { Patient and technique survival } \\
\text { (US): } 100 \% \text { (15 out of } 15 \\
\text { patients) }\end{array}$ \\
\hline $\begin{array}{l}\text { Pai et al., } \\
2016\end{array}$ & $\begin{array}{l}\text { 310: } 226 \\
\text { (US) vs. } 84 \\
\text { (PS) }\end{array}$ & Surgical & $\begin{array}{l}6-13 \text { days after } \\
\text { implantation }\end{array}$ & $\begin{array}{l}30 \pm 25 \\
\text { months }\end{array}$ & Not evaluated & $\begin{array}{l}\text { Peritonitis: } 1 / 65 \text { patient- } \\
\text {-month (US) vs. } 1 / 95 \text { patient- } \\
\text {-month (PS) [NS] }\end{array}$ & $\begin{array}{l}\text { Patient survival: } 80 \% \text { (US) vs. } \\
84 \% \text { (PS) [NS] } \\
\text { Technique survival: } 43.8 \% \\
\text { (US) vs. } 50.7 \% \text { (PS) [NS] }\end{array}$ \\
\hline $\begin{array}{l}\text { Nayak et } \\
\text { al, } 2018\end{array}$ & $\begin{array}{l}56: 32 \text { (US) } \\
\text { vs. } 24 \text { (PS) }\end{array}$ & Surgical & Start in 48h; APD. & 3 months & $\begin{array}{l}\text { Leakage: } 9.4 \% \text { (US) vs. 0\% (PS) } \\
\text { [NS] } \\
\text { Catheter migration: } 25 \% \text { (US) } \\
\text { vs. } 16.7 \% \text { (PS) [NS] }\end{array}$ & $\begin{array}{l}\text { Peritonitis: } 9.4 \% \text { (US) vs. } 0 \% \\
\text { (PS) [NS] }\end{array}$ & $\begin{array}{l}\text { Technique survival: } 90.6 \% \\
\text { (US) vs. } 95.8 \% \text { (PS) [NS] }\end{array}$ \\
\hline $\begin{array}{l}\text { Wojtaszek } \\
\text { et. al, } 2019\end{array}$ & $\begin{array}{l}\text { 129: } 35 \\
\text { (US) vs. } 94 \\
\text { (PS) }\end{array}$ & Surgical & $\begin{array}{l}\text { Urgent start (before } \\
2 \text { weeks); APD. }\end{array}$ & $\begin{array}{l}\text { Median of } \\
19 \text { months }\end{array}$ & $\begin{array}{l}\text { Leakage: } 11-14 \% \text { (US) vs. } \\
0-7 \% \text { (PS) [NS] } \\
\text { Catheter migration: } 3-9 \% \text { (US) } \\
\text { vs. 3-16\% (PS) [NS] }\end{array}$ & $\begin{array}{l}\text { Peritonitis: } 34 \% \text { (US) vs. } 33 \% \\
\text { (PS) [NS] }\end{array}$ & $\begin{array}{l}\text { Patient survival: Worse US in } \\
\text { the first } 3 \text { months, then } \\
\text { similar. } \\
\text { Technique survival: Excellent } \\
\text { in both [NS]. }\end{array}$ \\
\hline
\end{tabular}

APD - Automatic peritoneal dialysis; ESI - Exit site infection; HD - Hemodialysis; IPD - Intermittent peritoneal dialysis; NA - Not applied; NS - No significance; PD - Peritoneal Dialysis; PL - planned; US - Urgent start. 


\section{Table III}

Urgent-start PD versus Urgent-start HD

\begin{tabular}{|c|c|c|c|c|c|c|c|}
\hline Study & Patients (n) & Catheter placement & $\begin{array}{l}\text { Urgent-Start } \\
\text { Intervention }\end{array}$ & Follow-up & Mechanical Complications & Infectious Complications & Survival \\
\hline $\begin{array}{l}\text { Lobbedez } \\
\text { et al., } 2008\end{array}$ & $\begin{array}{l}\text { 60: } 34 \text { (US } \\
\text { PD) vs. } 26 \\
\text { (US HD) }\end{array}$ & Not specified & Median of 4 days & 12 months & $\begin{array}{l}\text { Leakage: } 5.8 \% \\
\text { Not specified for HD group. }\end{array}$ & $\begin{array}{l}\text { Survival free peritonitis: 55\% } \\
\text { at the end of follow up. } \\
\text { Not specified for HD group. }\end{array}$ & $\begin{array}{l}\text { Patient survival: } 83 \% \text { (US PD) } \\
\text { vs. } 79 \% \text { (US HD) [NS] } \\
\text { Technique survival: } 88 \% \text { (US } \\
\text { PD) }\end{array}$ \\
\hline $\begin{array}{l}\text { Koch et al, } \\
2012\end{array}$ & $\begin{array}{l}\text { 123: } 66 \text { (US } \\
\text { PD) vs. } 57 \\
\text { (US HD) }\end{array}$ & Laparoscopic & $12 \mathrm{~h}$ after; APD. & 6 months & $\begin{array}{l}\text { Catheter dysfunction: } 7.6 \% \\
\text { (US PD) vs. 5.3\% (US HD) [NS] }\end{array}$ & $\begin{array}{l}\text { Bacteremia: } 3 \% \text { (US PD) vs. } \\
21 \% \text { (US HD) ), } p=0.003 \\
\text { Peritonitis } 3 \% \\
\text { ESI: } 4,5 \%\end{array}$ & $\begin{array}{l}\text { Patient survival: } 70 \% \text { (US PD) } \\
\text { vs. } 58 \% \text { (US HD) [NS] }\end{array}$ \\
\hline $\begin{array}{l}\text { Jin et al., } \\
2016\end{array}$ & $\begin{array}{l}\text { 178: } 96 \text { (US } \\
\text { PD) vs. } 82 \\
\text { (US HD) }\end{array}$ & Surgical & $\begin{array}{l}\text { Urgent start (before } \\
2 \text { weeks) }\end{array}$ & 12 months & $\begin{array}{l}\text { Overall: } 3.1 \% \text { (US PD) vs. } \\
13.4 \% \text { (US HD), } p=0.011\end{array}$ & $\begin{array}{l}\text { Overall: } 2.1 \% \text { (US PD) vs. } 11 \% \\
\text { (US HD), } \mathrm{p}=0.014\end{array}$ & $\begin{array}{l}\text { Patient survival: } 92.1 \% \text { (US } \\
\text { PD) vs. } 93 \% \text { (US HD) [NS] }\end{array}$ \\
\hline
\end{tabular}

APD - Automatic peritoneal dialysis; ESI - Exit site infection; HD - Hemodialysis; IPD - Intermittent peritoneal dialysis; NA - Not applied; NS - No significance; PD - Peritoneal Dialysis; PL - planned; US - Urgent start.

should be made to improve the circuit of peritoneal catheter implantation toward an opportune schedule, sparing the vessels for later stages of CKD and respecting patient option. Even patients who starts HD with a temporary vascular access in emergency situations should be counselled as to the different dialysis modalities available, enabling the patient to make an informed subsequent decision.

Additionally, alternatives to hospital admission for urgent-start PD sessions (with inherent consumption of hospital resources and cost), nurse domiciliary visit and training or domiciliary hospitalization should be considered, as recently promoted in the management of chronic diseases and in hospital contracts processes.

To this end, protocols should be developed and the whole hospital community must be involved to make this possible.

\section{What does it take to make an Urgent-Start PD Program?}

In order to implement an urgent-start PD program (Table IV), a specialized team must always be on hand to insert a peritoneal catheter (it can be a nephrologist, a general surgeon, or even an intervention radiologist). Plus, a trained and specialized team is required for education and technique support throughout the initiation of the modality. Last but not the least, only selected candidates who have shown previous interest in PD should be forwarded to this process.

\section{CONCLUSION}

Urgent-start PD is a feasible and safe technique, with comparable results to HD and planned-start PD. In selected patients, and especially if PD was the patient's option for RRT, Urgent-Start PD should be considered as first-line therapy and a quality parameter in centers that are able to offer this kind of treatment. Trained staff and adequate infrastructure are required to run an Urgent-Start PD program, but

\section{Table IV}

Requirement for an Urgent-Start Peritoneal Dialysis Program

\begin{tabular}{l|l} 
Prompt catheter & - Critical step \\
placement (<48h) & - Nephrologist/ Surgeon/ Intervention radiologist (According to \\
Center availability) & - All techniques are suitable (Percutaneous/ Surgical/ \\
& Laparoscopic)
\end{tabular}

overall a strategic plan is needed to promote value-based healthcare. We propose a simple algorithm that could be implemented and adapted in other centers (Figure 2).

Additional studies should be performed in terms of dialysis efficiency and in terms of urgent-start dialysis prescription.

This proposal is meant to signal an updated approach to patient-centered integrated CKD care and moreover quality improvement management of dialysis access in integrated dialysis units.

Disclosure of potential conflicts of interest: none declared 
Figure 2

Algorithm Urgent-Start Peritoneal Dialysis

\section{URGENT-START DIALYSIS}

Emergent criteria for dialysis?

(Severe hyperkalemia or metabolic acidosis; Hypervolemia; Severe uremia)

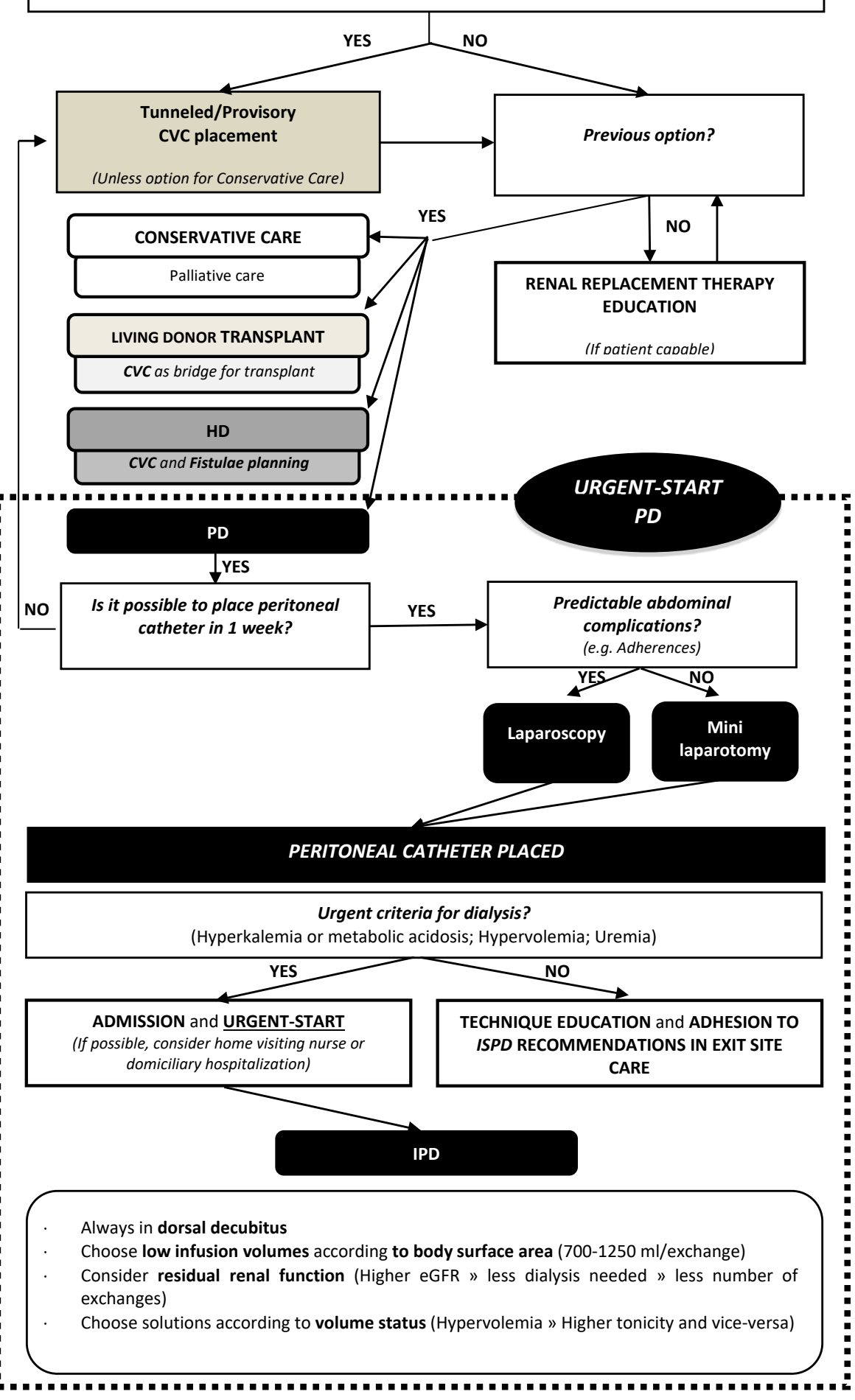




\section{References}

1. Saran R, Robinson B, Abbott KC, Agodoa LYC, Bragg-Gresham J, Balkrishnan R, et al. US Renal Data System 2018 Annual Data Report: Epidemiology of Kidney Disease in the United States. Am J Kidney Dis. 2019;73(3s1):A7-A8.

2. Bbg01.com. (2019). [online] Available at: http://www.bbg01.com/cdn/rsc/spnefro/gabreg/305/ RelatriosAnuais2018.pdf [Accessed 10 Sep. 2019

3. Liyanage T, Ninomiya T, Jha V, Neal B, Patrice HM, Okpechi I, et al. Worldwide access to treatment for end-stage kidney disease: a systematic review. Lancet. 2015;385(9981):1975-82.

4. Li PK, Kwong VW. Current challenges and ppportunities in PD. Semin Nephrol. 2017;37(1):2-9.

5. Blake PG, Quinn RR, Oliver MJ. Peritoneal dialysis and the process of modality selection. Perit Dial Int. 2013;33(3):233-41

6. Perl J, Wald R, McFarlane P, Bargman JM, Vonesh E, Na Y, et al. Hemodialysis vascular access modifies the association between dialysis modality and survival. J Am Soc Nephrol. 2011;22(6):1113$-21$.

7. Lorenzo V, Martn M, Rufino M, Hernandez D, Torres A, Ayus JC. Predialysis nephrologic care and a functioning arteriovenous fistula at entry are associated with better survival in incident hemodialysis patients: an observational cohort study. Am J Kidney Dis. 2004;43(6):999-1007.

8. Ghaffari A. Urgent-start peritoneal dialysis: a quality improvement report. Am J Kidney Dis. 2012;59(3):400-8.

9. Jin $\mathrm{H}$, Fang W, Zhu M, Yu Z, Fang $\mathrm{Y}$, Yan $\mathrm{H}$, et al. Urgent-start peritoneal dialysis and hemodialysis in ESRD patients: complications and outcomes. PLoS One. 2016;11(11):e0166181.

10. Koch M, Kohnle M, Trapp R, Haastert B, Rump LC, Aker S. Comparable outcome of acute unplanned peritoneal dialysis and haemodialysis. Nephrol Dial Transplant. 2012;27(1):375-80.

11. Lobbedez T, Lecouf A, Ficheux M, Henri P, Hurault de Ligny B, Ryckelynck JP. Is rapid initiation of peritoneal dialysis feasible in unplanned dialysis patients? A single-centre experience. Nephro Dial Transplant. 2008;23(10):3290-4.

12. Alkatheeri AM, Blake PG, Gray D, Jain AK. Success of urgent-start peritoneal dialysis in a large canadian renal program. Perit Dial Int. 2016;36(2):171-6.

13. Banli O, Altun H, Oztemel A. Early start of CAPD with the Seldinger technique. Perit Dial Int 2005;25(6):556-9.

14. Bitencourt Dias D, Mendes ML, Burgugi Banin V, Barretti P, Ponce D. Urgent-start peritoneal dialysis: the first year of Brazilian experience. Blood Purif. 2017;44(4):283-7.
15. Jo $\mathrm{YI}$, Shin SK, Lee JH, Song JO, Park JH. Immediate initiation of CAPD following percutaneous catheter placement without break-in procedure. Perit Dial Int. 2007;27(2):179-83.

16. Song JH, Kim GA, Lee SW, Kim MJ. Clinical outcomes of immediate full-volume exchange one year after peritoneal catheter implantation for CAPD. Perit Dial Int. 2000;20(2):194-9.

17. Wang C, Fu X, Yang Y, Deng J, Zhang HQ, Deng HM, et al. A comparison between intermittent peritoneal dialysis and automatic peritoneal dialysis on urgent peritoneal dialysis. Am J Nephrol. 2017;45(6):540-8

18. Wong LP, Li NC, Kansal S, Lacson E, Jr., Maddux F, Kessler J, et al. Urgent peritoneal dialysis starts for ESRD: initial multicenter experiences in the United States. Am J Kidney Dis. 2016;68(3):500-2.

19. Xu D, Liu T, Dong J. Urgent-start peritoneal dialysis complications: prevalence and risk factors. Am J Kidney Dis. 2017;70(1):102-10

20. Nayak KS, Subhramanyam SV, Pavankumar N, Antony S, Sarfaraz Khan MA. Emergent start peritoneal dialysis for end-stage renal disease: outcomes and advantages. Blood Purif. 2018;45(4):313-9 .

21. Pai MF, Yang JY, Chen HY, Hsu SP, Chiu YL, Wu HY, et al. Comparing long-term outcomes between early and delayed initiation of peritoneal dialysis following catheter implantation. Ren Fail. 2016;38(6):875-81.

22. Povlsen JV, Ivarsen P. How to start the late referred ESRD patient urgently on chronic APD. Nephrol Dial Transplant. 2006;21(2):ii56-9.

23. Wojtaszek E, Grzejszczak A, Grygiel K, Malyszko J, Matuszkiewicz-Rowinska J. Urgent-start peritoneal dialysis as a bridge to definitive chronic renal replacement therapy: short- and long-term outcomes. Front Physiol. 2018;9:1830.

24. Yang YF, Wang HJ, Yeh CC, Lin HH, Huang CC. Early initiation of continuous ambulatory peritoneal dialysis in patients undergoing surgical implantation of Tenckhoff catheters. Perit Dial Int. 2011;31(5):551-7

\section{Correspondence to:}

Joana Manuel Silva Fernandes Lopes Tavares

Serviço de Nefrologia, Centro Hospitalar Universitário do Porto

Largo do Prof. Abel Salazar 4099-001 Porto, Portugal

E-mail: joanatavares91@gmail.com 